\title{
O Comportamento Ético das Corporações Afeta as Relações com suas Marcas? Influência da Percepção Ética do Consumidor (PEC) na Confiança, no Comprometimento e na Leal- dade dos Consumidores
}

\section{Does Corporate Ethical Behavior Affect Relationships with their Brands? Influence of Ethical Consumer Perception on Consumers' Trust, Commitment and Loyalty}

\author{
Renata de Sousa da Silva Tolentino \\ Universidade FUMEC - Brasil \\ rsousa@fumec.br \\ ORCID: 0000-0002-8284-7509 \\ Cid Gonçalves Filho \\ Universidade FUMEC - Brasil \\ cid@fumec.br \\ ORCID: 0000-0002-2454-9546 \\ Jefferson Lopes La Falce \\ Universidade FUMEC - Brasil \\ jefferson.la.falce@gmail.com \\ ORCID: 0000-0002-3293-2908
}

Submetido em 24/12/2018; Aprovado em 03/06/2019

\section{Resumo}

Objetivos: 0 objetivo deste estudo foi verificar as influências da percepção ética, da confiança, do comprometimento na lealdade dos consumidores. Metodologia: Foram realizados grupos de foco que visaram explorar as dimensões da PEC no contexto do mercado em estudo. Em seguida, foi realizado um experimento de laboratório com 270 respondentes, visando verificar como comportamentos éticos de uma corporação afetam a confiança, o comprometimento e a lealdade de seus consumidores. Um modelo hipotético integrativo foi testado por meio de modelagem de equações estruturais. Principais resultados: Os resultados demonstraram que o modelo foi capaz de explicar que a percepção ética do consumidor influencia a confiança, o comprometimento e a lealdade dos consumidores em relação às empresas. 0 comportamento ético afetou fortemente a confiança e, significativamente e em menor impacto, o comprometimento. Os resultados indicam que o trinômio ética-confiança-comprometimento age de forma integrada em situações éticas, fomentando a lealdade. Contribuições acadêmicas: A confiança é um sentimento de segurança demonstrado pelo cliente em sua interação com a marca e baseia-se nas percepções de que a marca é confiável e responsável pelos interesses e pelo bem-estar do consumidor. Nesse sentido, as principais contribuições deste trabalho são a inclusão da ética na cadeia nomológica da lealdade das empresas, bem como a indicação de seus impactos na confiança e no comprometimento. Contribuições práticas: A confiança é significativamente afetada pela percepção ética, apresentando aos gerentes uma afirmação de que suas ações são acompanhadas de perto pelos consumidores, e eles devem adotar medidas rápidas para conter os eventuais problemas de uma percepção não ética. A empresa deve ser sincera com o cliente, responder às falhas rapidamente. Atitudes éticas são percebidas pelos consumidores como avanços e geram confiança e comprometimento deles, portanto as marcas devem estar atentas quanto à execução e divulgação de suas ações éticas em relação aos consumidores, ao meio ambiente e à corrupção.

Palavras-chave: Ética empresarial; Comportamento do consumidor; Lealdade; Confiança; Comprometimento.

\section{Abstract}

Objectives: The purpose of this study was to verify the influences of Ethical Perception in the loyalty of consumers was developed this study. Methodology: Focus groups were conducted to explore the dimensions of PEC in the context of the market under study. Then, a laboratory experiment was carried 
out with 270 respondents, in order to verify how ethical behavior of a corporation affects the confidence, commitment and loyalty of its consumers. An integrative hypothetical model was tested through structural equation modeling Main results: The results showed that the model was able to explain $70.3 \%$ of loyalty. Ethical behavior strongly affected the trust, and significantly, and in less impact the commitment. The results indicate that the trinomial ethics-trust-commitment works in an integrated way in ethical situations fostering loyalty. Academic contributions: Considering stimuli linked to ethics, it is observed that they are generators (or reducers) in particular of trust. Trust is a sense of security held by the customer in their interaction with the brand and is based on the perceptions that the brand is trustworthy and responsible for the interests and well-being of the consumer. In this sense, the main contributions of this work are the inclusion of ethics in the nomological chain of business loyalty, as well as indicating its impact on trust and commitment. Practical contributions: Trust is significantly affected by ethical perceptions, presenting an assertion to managers that their actions are accompanied by consumers and they must take swift action to contain any problems of unethical perception, the company must be honest with the client, respond to glitches quickly. Ethical attitudes are perceived by consumers as advancements and generate confidence and commitment of the same, so brands must be attentive in the execution and dissemination of their ethical actions towards consumers, the environment and corruption.

Keywords: business ethics, consumer behavior, loyalty, trust, commitment

\section{Introdução}

Nos últimos anos, a preocupação dos consumidores com o comportamento ético de empresas fez com que estas passassem a procurar compreender como essa relação poderia impactar a decisão de compra (Singhapakdi, Gopinath, Marta \& Carter, 2008). Desde a década de 1990, observa-se um crescimento no número de pesquisas realizadas no campo da ética e de valores de responsabilidade social (Wilkie \& Moore, 2012). A ética é atualmente uma das mais importantes demandas dos consumidores, que vivenciam, em diversos países, dilemas éticos que afetam a classe política, bem como as empresas. Quando uma empresa tem um comportamento ético, acredita-se que as impressões dos consumidores sobre sua marca corporativa e seus produtos e serviços podem ser afetadas (Hamidizadeh, Alavije \& Rezaee, 2014).

Por outro lado, a relação entre consumidores e marcas (Consumer-Brand Relationships - CBR) é um tema de expressão na literatura de marketing contemporânea (Keller, 1993, 2001). Blackston (1992) foi um dos primeiros autores a sugerir estudos sobre consumidores e suas relações com as marca. Para Fetscherin e Heilman (2015), o relacionamento consumidor-marca pode ser entendido por meio da Teoria do Relacionamento Consumidor-Marca, denominada Consumer-Brand Relationships $(C B R)$. 0 processo pode ser entendido pelas interações repetidas, ocorridas entre uma marca e um consumidor. Estudos como o de Fournier (1998) têm evidenciado importantes consequências da qualidade do relacionamento consumidor-marca para as organizações, tais como tolerância, perdão, viés de atributos, parceria e continuidade do relacionamento, evidenciando a relevância do desenvolvimento desse relacionamento no longo prazo. Já como resultado de um adequado relacionamento consumidormarca, a lealdade dos consumidores é considerada um relevante comportamento, por suas consequências na lucratividade, bem como no incremento do valor vitalício dos consumidores.

Em pesquisa realizada nas bases SPELL e EBSCO, com as palavras-chave "comportamento ético" e "consumidores", e com essas palavras também em inglês, verificou-se a inexistência de estudos que exploram os impactos do comportamento ético das empresas nas relações entre consumidores e marcas, bem como a exploração das dimensões éticas de empresas em contexto de países em desenvolvimento e emergentes, sendo que, no sentido de suprir essa falta, foi elaborado este estudo.

Uma quantidade significativa de pesquisas investigou o impacto do comportamento não ético de uma empresa sobre as atitudes dos consumidores e como uma firma pode se recuperar após uma crise da marca (Kashmiri et al., 2017). Por outro lado, questões envolvendo marketing, ética e responsabilidade social são, em geral, controversas, e anos de pesquisas têm apresentado visões desafiadoras e conflitantes, para pesquisadores e gestores, sobre a sua importância para as atividades de marketing (Carrigan \& Attalla, 2001).

Sierra et al. (2017) argumentam que, apesar do número representativo de estudos que relacionam ética com marketing, a pesquisa sobre ética na área de branding ainda é escassa. Entre essas pesquisas, assinala-se a existência de estudos na interseção da ética empresarial e da gestão de marca corporativa, 
tais como os de Folkes e Kamins (1999), Fan (2005), Rindell et al. (2011), Hutchinson et al. (2013) e Sierra et al. (2017). Nesse sentido, apesar da relevância justificada do campo do branding e do relacionamento consumidor-marca, pesquisas que vinculam gestão da marca corporativa com ética empresarial são de âmbito mais conceitual ou limitadas a aspectos restritos da gestão de marcas (Sierra et al., 2017).

Por outro lado, o conceito e a escala PEC, que tem sido aplicada e validada na literatura, foram desenvolvidos sob a ótica de países desenvolvidos (Brunk, 2010; 2012), nos quais a ética empresarial é uma prática mais comum e onde consumidores percebem mais seus direitos, o que sugere dimensões contextuais percebidas pelos consumidores de forma alinhada às práticas realizadas pelas empresas com as quais se relacionam. Diferenças entre percepção ética e nível ético em países desenvolvidos e em desenvolvimento/emergentes têm sido recorrentes na literatura, porém a operacionalização e o estudo empírico dessas diferenças pode requerer aprofundamento (Arli \& Lasmono, 2009). Leigh (2014) argumenta que a economia mundial cada dia está mais dependente de países emergentes, tais como China e Brasil, porém as realidades nesses países em desenvolvimento podem incluir aspectos éticos específicos, tais como maiores níveis de maus-tratos no trabalho, corrupção, agitação política, informações incertas de terceiros e mudanças regulatórias locais, sugerindo que questões éticas poderiam apresentar novas facetas por causa desses novos contextos e ambiente de negócios. Nesse sentido, este trabalho busca contribuir para o aprimoramento da escala e a construção teórica da Consumer Perceived Ethicality e sua generalização.

Desse modo, visando propor uma melhor compreensão da formação do relacionamento consumidor-marca e suas relações com a percepção ética do consumidor, foi proposta esta pesquisa. Nesse sentido, buscou-se evidenciar um modelo integrativo de estágios de CBR, tendo como variável independente a percepção ética do consumidor em relação às empresas de modo a atingir o seguinte objetivo: verificar as influências da percepção ética, da confiança, do comprometimento e da lealdade dos consumidores. Tal integração ética-marcas (CPE-CBR) foi estruturada em uma cadeia nomológica de antecedentes e consequentes do processo de desenvolvimento de relacionamentos entre consumidores e marcas, visando ampliar as bases de compreensão do fenômeno.

\section{Revisão da literatura}

Alguns especialistas em ética de negócios tratam a Ética como um ramo da Filosofia, envolvendo dois tipos de princípios morais: Deontologia, parte da Filosofia que trata dos princípios, fundamentos e sistemas de moral; estudo dos deveres; e Teleologia, teoria das causas finais; conjunto de especulações que têm em vista o conhecimento da finalidade, encarada de modo abstrato, pela consideração dos seres (Shanahan \& Hyman, 2003). Embora existam muitos estudos sobre filosofia desenvolvidos por pesquisadores na exploração da natureza do julgamento moral (Callicott \& Frodeman, 2009; Dutt \& Wilber, 2010; Pojman \& Fieser, 2012; Quinn, 2013 e Cuza, 2012), deontologia e teleologia são dois conceitos considerados dominantes encontrados na literatura (Brunk, 2010).

Nos últimos anos, a investigação, no domínio do consumidor, sobre a percepção de práticas éticas e corporativas, responsabilidade social da empresa e seu impacto sobre o desempenho dos negócios tem sido desenvolvida, por causa de sua possível alavancagem de vantagem competitiva dos valores éticos (Chabowski, Mena \& Gonzalez-Padron, 2011). Em relação ao consumidor, há tendência emergente do consumo ético, e os consumidores não só aplicam os princípios responsáveis no comportamento de compra, como também procuram informações sobre a reputação das empresas com as quais querem se relacionar (Brunk, 2010). Em contrapartida, no mercado, existem empresas que se esforçam para o desenvolvimento de reputação ética, empregando estratégias de responsabilidade social corporativa, desenvolvimento de códigos de ética ou comunicando valores, tais como honestidade e integridade.

Brunk (2010) foi uma das primeiras investigadoras que visaram nomear as raízes da percepção ética dos consumidores, isto é, entender qual o envolvimento dos consumidores em atividades quando a questão da ética é levantada. "A Percepção Ética do Consumidor (PEC) pode ser definida como a percepção agregada dos consumidores da moralidade de um sujeito (empresa, marca, produto ou serviço)" (Brunk \& Blumelhuber, 2011, p. 134). Nesse sentido, uma série de estudos tem sido realizada de forma a contextualizar e explorar esse fenômeno (Besiou, Mark \& Wassenhove, 2013; Das, Agarwal, Malhotra \& Varshneya, 2019; Grohmann \& Bodur, 2015). Esses autores investigaram a natureza da percepção dos consumidores, identificando os grandes grupos de atividades empresariais que são notadas como 
éticas e não éticas, e também quais seriam os fatores importantes para definir a empresa ética. Um segundo estudo realizado por Brunk (2012) desenvolveu uma escala, identificando as variáveis exatas que podem ser usadas na medição de ética percebida pelos consumidores.

No processo de exploração do construto e sua operacionalização, Brunk e Blumelhuber (2011) utilizaram metodologias de pesquisa qualitativa, com o objetivo de explorar as percepções do consumidor sobre ética empresarial. Esses pesquisadores identificaram que todas as respostas podem ser enquadradas em seis categorias principais, em conjunto com 36 subcategorias que descrevem a possível origem da percepção ética do consumidor. De acordo com a pesquisa, os clientes tendem a avaliar a ética de uma empresa com base nestes seis fatores: consumidores, empregados, meio ambiente, comunidade local e da economia, comunidade de negócios e comunidade no exterior. Na sequência, Brunk (2012) realizou três estudos complementares, utilizando técnicas qualitativas e quantitativas, que se encerraram com especificação, conceituação e validação da escala de Percepção Ética do Consumidor (PEC).

\subsection{Relacionamento consumidor-marca}

Segundo Blackston (1992), o relacionamento consumidor-marca é uma extensão lógica da personalidade da marca, assemelhando-se ao relacionamento entre as pessoas. Fournier (1998) sugere que o relacionamento da marca é um vínculo emocional resultante da interação entre os consumidores e as marcas. Nesse sentido, essa relação é interpretada como uma relação de amor, comparando o relacionamento consumidor-marca com o relacionamento humano. Essas relações entre o consumidor e as marcas não somente existem, como também seriam desenvolvidas ao longo do tempo, através de todas as experiências e interações (Bowden, 2009), levando as firmas a executar várias tarefas para construílas e mantê-las (Parvatiyar \& Sheth, 2000). A relação consumidor-marca pode ser pensada como um resultado do processo interativo sequencial entre o consumidor e uma marca personificada em uma perspectiva de longo prazo, em vez de um padrão simples de recompra com base na satisfação dos consumidores com um produto ou um serviço. Sreejesh (2014) e Sreejesh e Roy (2015) desenvolveram estudos em que utilizaram uma abordagem mista de métodos que exploraram a integração de construções atitudinais e comportamentais e conceituais, visando à proposição de um modelo integrado do Relacionamento Consumidor-Marca. Nesses estudos, foi realizada uma fase qualitativa para compreender as dimensões do CBR, sob a perspectiva da Grounded Theory (Strauss \& Corbin, 1994). 0 modelo proposto é exibido na Figura 1.

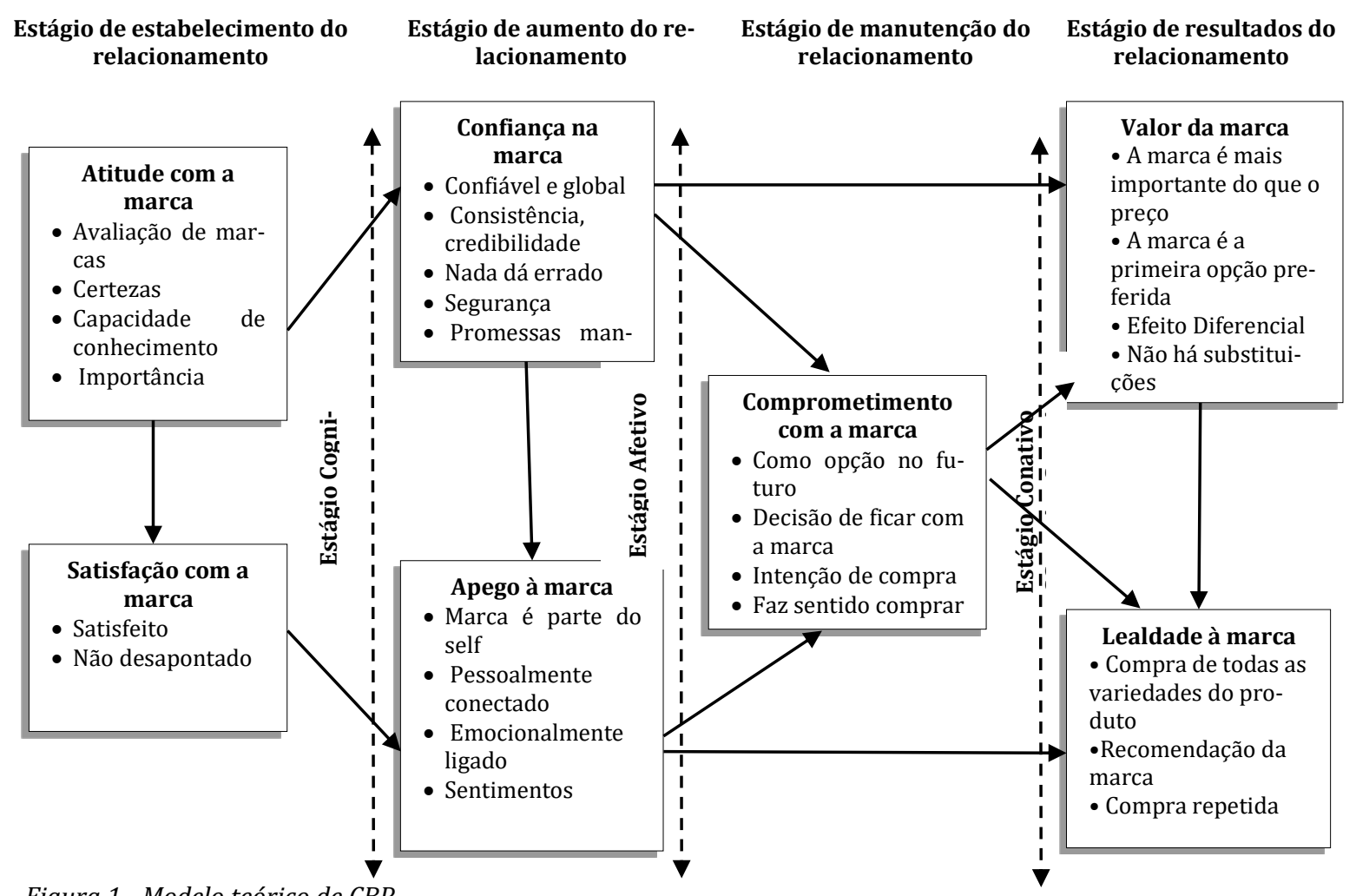

Figura 1 - Modelo teórico de CBR

Fonte: Sreejesh \& Roy (2015). 
A fase atitudinal do CBR (Consumer-Brand Relationships) tem três estágios fundamentais: cognitivo, afetivo e conativo (Oliver, 1999). No estágio cognitivo, os consumidores desenvolvem a força de atitude por meio da comparação da marca e de suas alternativas, com base em experiências passadas e/ou conhecimento relacionados à oferta, aos atributos da marca, ao seu desempenho ou a informações baseadas na experiência atual sobre a marca (Sreejesh, 2014; Oliver, 1999; Blackston, 1992). A segunda etapa do $C B R$ consiste em um estágio afetivo, no qual ocorre um aprofundamento da relação. Segundo Sreejesh (2014) e Oliver (1999), neste estágio o relacionamento desenvolve-se a partir de fortes atitudes favoráveis à marca e sua avaliação global. Esta fase ocorre quando a marca oferece recursos que influirão no self e se desenvolve por meio da confiança na marca ou da sensação de consistência em relação ao desempenho da marca. Já o terceiro estágio, ou estágio conativo, refere-se à manutenção do relacionamento, no qual o consumidor desenvolve uma situação de comprometimento com a marca, decidindo continuar com aquela marca, bem como vendo-a como opção futura de compra. Por fim, a fase de resultados do relacionamento ou comportamental consiste em desenvolvimento do brand equity (valor da marca para o consumidor), lealdade à marca, intenção de compra e boca a boca positivo.

\subsection{Comportamento ético das organizações e o comportamento do consumidor}

Historicamente, o consumo ético era visto muito como o comportamento de um grupo relativamente pequeno de consumidores de princípios (Shaw, 2007), enquanto as marcas éticas (por exemplo: The Body Shop) eram fáceis de identificar. No entanto, esse número de marcas éticas, ou seja, marcas que apresentam um comportamento ético em relação ao meio ambiente, aos consumidores e em relação à corrupção, aumentou, em conjunto com a facilidade de acesso aos dados relativos a produtos éticos (O'Connor, 2014). O conceito de fair trade, por exemplo, tem desenvolvido a partir de um foco organizacional de produtores para incorporar questões de justiça social mais amplas (Becchetti \& Costantino, 2010).

Muitas definições de consumo ético abrangem referências a questões ambientais e reciclagem (Laroche, Bergeron \& Barbaro-Forleo, 2001). Isso pode se dever ao fato de que a reciclagem de resíduos domésticos está se tornando um comportamento normal e diário para muitas pessoas, em parte devido a políticas e programas de reciclagem em muitos países. São também frequentes na literatura, estudos sobre as relações do consumo ético abrangendo justiça social e questões de direitos humanos (Auger, Devinney, Louviere \& Burke, 2010; Becchetti \& Costantino, 2010), muitas vezes com uma ênfase particular envolvendo a na exploração dos trabalhadores. Além dessas questões dominantes, as definições mais abrangentes concentram-se em bem-estar animal (Megicks, Memery \& Williams, 2008) e iniciativas da comunidade local (Carrigan, Moraes \& Leek, 2011). Por fim, a literatura associa consumo ético à recusa consciente para não comprar produtos (Carrigan, Szmigin \& Wright, 2004).

Teoricamente, a distinção entre a escolha de um produto ético e a recusa a comprar um produto não ético é um passo importante. Carrigan e Attalla (2001) explicam que as ações éticas e antiéticas têm uma influência assimétrica sobre os consumidores em punir o comportamento antiético (boicote), mas não necessariamente em recompensar o comportamento ético (compra de mais alternativas).

\section{Proposição das hipóteses}

Uma empresa que tem um comportamento ético percebe que as impressões dos clientes sobre seus produtos e serviços podem mudar radicalmente (Brunk \& Blumelhuber, 2011). Consumidores podem deixar de comprar ou utilizar produtos de determinadas marcas, quando estas se envolvem em questões éticas. Os clientes passam a ver a empresa como responsável para a sociedade, e isso vai reforçar a personalidade da marca, afetando a confiança, o comprometimento e o apego para com ela (Brunk \& Blumelhuber, 2011). Para Wu e Chen (2015), os consumidores adquirem confiança em uma marca quando sabem que a companhia está se comportando eticamente. Às vezes, uma única ação pode definir a percepção ética de um consumidor de uma companhia ou marca, como no caso do consumidor com percepção negativa da marca Starbucks (Brunk \& Blumelhuber, 2011).

A percepção ética de um consumidor (PEC - Consumer Perceived Ethicality - CPE) é influenciada pelo comportamento ético de uma companhia ou marca em relação a consumidor; empregados, ambiente; comunidade ou economia local; comunidade de negócios e comunidade internacional (Brunk, 2010), o que pode gerar um impacto positivo na confiança na marca (Argoon \& Rambocas, 2011; Lin \& Chuang, 2016; Chinomona, 2013). Para Schmalz e Orth (2012), o apego à marca é um importante determinante das respostas do consumidor à ética praticada pela firma, corroborando Young e Hee (2006). 
0 apego à marca tem um viés sobre os julgamentos da ética da firma.

A relação entre a ética percebida e a confiança na marca apresenta-se significativamente positiva em estudos com consumidores de mercados presenciais e virtuais (Singh, Iglesias \& Foguet, 2012; Nga \& Soo, 2013). Os resultados sugerem que existe uma relação positiva entre a ética percebida de uma marca e a confiança na marca e a influência da marca.

0 comprometimento do consumidor em relação à marca é identificado como um moderador de efeitos de informação negativa ou não ética (Ingram \& Skinner, 2005). Para Morgan e Hunt (1994), a confiança e o comprometimento são variáveis que encorajam os parceiros em uma relação a trabalhar preservando essa relação, evitando novas relações com outros parceiros e reduzindo a percepção de risco no ambiente de mercado. Desse modo, as seguintes hipóteses foram propostas:

H1: A percepção ética do consumidor tem um efeito positivo sobre confiança na marca.

H2: A percepção ética do consumidor tem um efeito positivo no comprometimento

Esch, Langner, Schmitt e Geus (2006) argumentam que os estudos empíricos que relacionam a satisfação com a marca, a confiança na marca e o apego à marca também observam suas relações com a consciência e a imagem da marca. Essa proposta corrobora o estudo de Sreejesh e Roy (2015), que verificaram empiricamente essas relações. Resultados similares foram reportados por Sondoh et al. (2007) na indústria de cosméticos.

Na literatura de marketing, a confiança é considerada como um elemento essencial para o desenvolvimento do apego à marca e tem sido reconhecida como uma ferramenta importante, que busca melhorar o desempenho da marca (Carroll \& Ahuvia, 2006). Para Chiu, Huang e Yen (2010), os consumidores que confiam em uma marca estão dispostos a melhorar e manter um vínculo afetivo com ela. Dib e Alhaddad (2014) verificaram empiricamente o impacto da confiança no brand equity em mercado de telefones celulares, corroborando resultados de pesquisas de Ganesan (1994) e Morgan e Hunt (1994). Ainda cabe ressaltar que a confiança do consumidor é frequentemente apontada como um dos elementos essenciais para que os clientes/consumidores desenvolvam mais que um sentimento de comprometimento com esse parceiro (Bloemer \& Oderkerken-Schröder, 2002; Chaudhuri \& Hoolbrook, 2002).

A confiança na marca pode influenciar o comprometimento quando há informações de percepção ética da marca, segundo Raju, Unnava e Montgomery (2009). Por outro lado, o apego à marca influencia o comportamento do consumidor no que ser refere ao seu comprometimento, segundo observam Dennis et al. (2016) e Zhang, Zhou e Zhou (2013). Nesse sentido, a seguinte hipótese foi proposta:

H3: A confiança na marca tem um efeito positivo sobre o comprometimento com a marca.

0 terceiro estágio, de manutenção ou conativo, proposto por Sreejesh \& Roy (2015), está associado ao desenvolvimento do comprometimento dos consumidores com as marcas. Acredita-se que maiores níveis de comprometimento estejam associados com a percepção de ganhos futuros, com a identificação do parceiro, com o desejo limitado de buscar outros parceiros, com a quantidade de esforço, de investimento feito no relacionamento e com a responsabilidade assumida pelo indivíduo (Knapp \& Taylor, 1994).

Keller $(1993,2001)$ argumenta que a construção do brand equity passa pelo comprometimento do consumidor com a marca, o que corrobora o estudo de Sreejesh (2014), no qual se verificaram pesos significativos do comprometimento no brand equity. Nesse sentido, Dick e Basu (1994), bem como Hess e Story (2005), consideram que consumidores comprometidos com uma marca tendem a desenvolver atitudes positivas com relação às marcas (Jang et al., 2014).

A relação entre o comprometimento com a marca, a lealdade e a intenção de compra é muito importante para confirmar a percepção do consumidor sobre um serviço ou produto (McAlexander, Kim \& Roberts, 2015; Iglesias, Singh \& Batista-Foguet, 2011). Identificar combinações entre vários tipos de comprometimento e benefícios, para induzir e aprimorar comportamentos de fidelidade, como retenção de clientes e confirmação de venda, consiste em evidências empíricas em mercados de produtos e serviços (Hur, Park \& Kim, 2010). Nesse sentido, a seguinte hipótese foi proposta:

H4: Comprometimento com a marca tem um efeito positivo sobre a lealdade à marca.

A seguir será apresentado o caminho metodológico da pesquisa.

Figura 2 - Modelo Hipotético de Pesquisa 
Estágio de Estabelecimento de Relacionamento
Estágio de Aumento do Relacionamento
Estágio de Manutenção do Relacionamento
Estágio de Resultado do Relacionamento

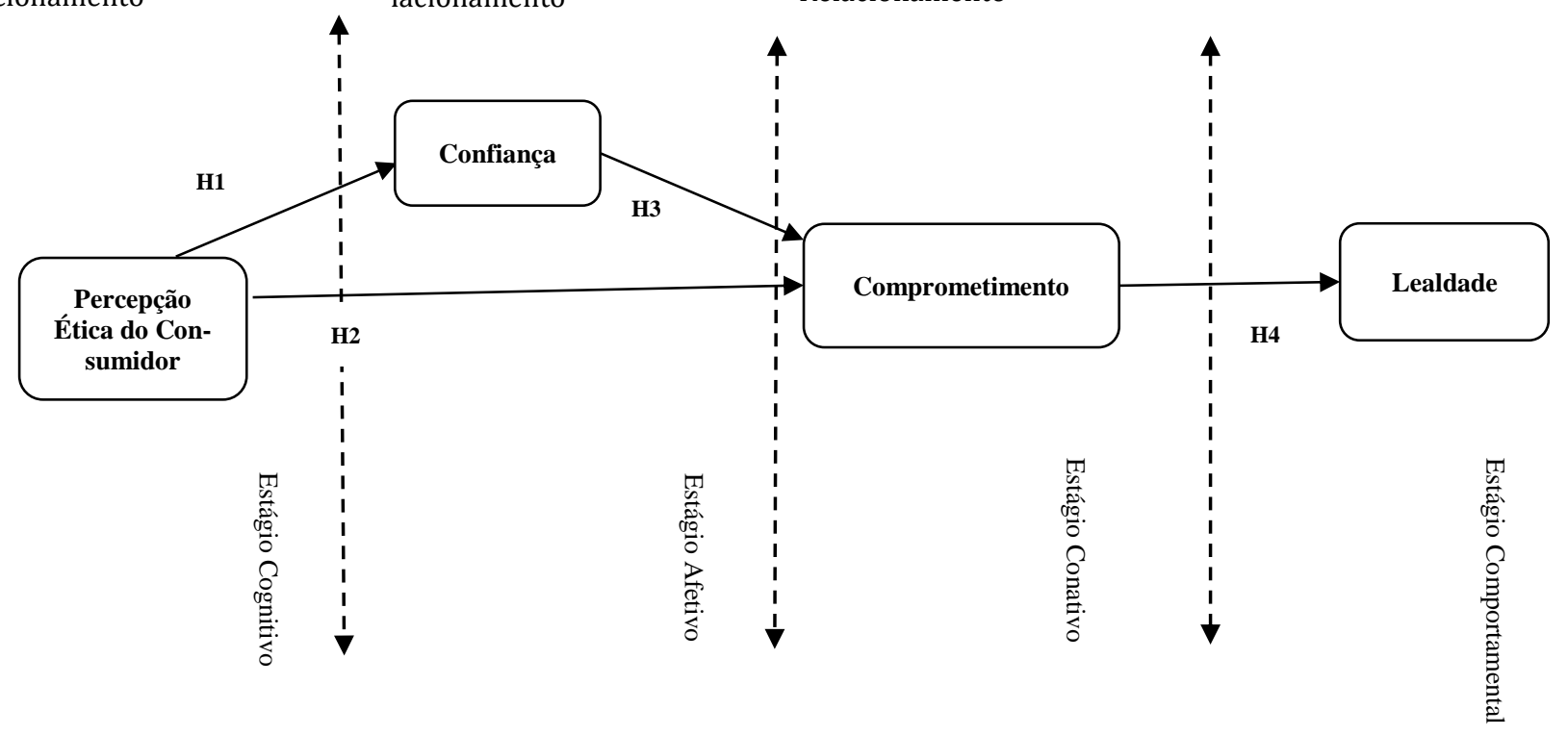

Fonte: Elaborada pelos autores.

\section{Metodologia}

Para atingir os objetivos da pesquisa, foram realizados dois grupos de foco em fevereiro de 2017, com 10 participantes cada um. Esses participantes eram, em um grupo, consumidores de diversas empresas de smartphones e profissionais de tecnologia da informação pertencentes à geração Y e, em outro grupo, eram consumidores de smartphones e profissionais de estética, também pertencentes à geração Y. Observou-se que um total de 10 participantes seria ideal para a discussão da escala proposta e que também seria confortável para eles responderem. A escala foi traduzida por meio de roteiro semiestruturado de 15 questões, em que foram exploradas as relações éticas entre empresas e consumidores. Os grupos foram extremamente importantes para a pesquisa, pois validaram a escala de Percepção Ética dos Consumidores de Brunk (2012), porém revelaram que, no Brasil, existem duas outras possíveis dimensões adicionais de Ética Empresarial, não citadas na literatura e em fontes internacionais: o respeito ao consumidor e a corrupção. Neste sentido, essas questões foram adicionadas à escala de Brunk (2012).

Posteriormente, foi realizado um experimento no mês de março de 2017, com 270 participantes possuidores de smartphones em uma grande capital brasileira. Para tanto, foi dado um incentivo que correspondia a um vale-lanche. Os respondentes, estudantes universitários de uma grande universidade, de diversos cursos superiores, responderam ao questionário resultante do grupo de foco, com o incremento das situações hipotéticas com o comportamento ético e não ético das empresas.

0 experimento foi realizado em ambiente de laboratório, um contexto artificial para experimentação no qual o pesquisador constrói as condições desejadas, pois oferece um elevado grau de controle, na medida em que isola o experimento de forma a ser cuidadosamente monitorado (Malhotra, 2001). Nesse sentido, um procedimento experimental foi inserido, sendo que os respondentes receberam um questionário contendo duas partes, uma que relatava como notícia (estímulo) o comportamento ético da fabricante de smartphone e outra uma descrição que relatava comportamento ético, abordando todas as dimensões da escala de Brunk (2012). 0 experimento de laboratório ocorreu de acordo com procedimentos sugeridos por Malhotra (2001). A descrição da notícia (estímulo) de empresa não ética é mostrada a seguir:

"Recentemente, foi verificado que a marca de seu celular possui uma grande fábrica no exterior, na qual os trabalhadores recebem um tratamento especial e salários superiores aos das demais empresas, com jornadas de trabalho adequadas. O nível de retenção de funcionários e satisfação é elevado. A fábrica trata todos os seus resíduos, sendo muito responsável com o ambiente. Além disso, ela busca apoiar causas sociais, tais como hospitais, creches e escolas. O serviço a clientes no pós-venda é excelente, sendo que a empresa troca ou conserta 
produtos rapidamente, respeitando seus consumidores. A empresa é ética e não aceita atos de corrupção. Considerando esses fatos sobre a empresa que fabrica seu celular, responda às questões abaixo".

Para operacionalizar a mensuração das variáveis, foram adaptadas a esse modelo as escalas confiança na marca ou Brand Trust, com quatro itens (He, Li \& Harris, 2012; Chinomona, 2013); comprometimento com a marca ou Brand Commitment, com quatro itens (Eisingerich \& Rubera, 2010); lealdade à marca ou Brand Loyalty, com sete itens (Petzer, Mostert, Kruger \& Kuhn, 2014), e escala ética, com seis itens de Brunk (2012), acrescida de duas questões obtidas na fase qualitativa e que contextualizam ética empresarial no cenário brasileiro. As escalas utilizadas para este estudo foram de 11 pontos, visando facilitar o tratamento como variáveis quantitativas contínuas, todas do tipo Likert.

Para a análise de dados, foi realizada uma análise exploratória que busca revelar características do banco de dados e demonstrar se ocorrem violações nos pressupostos estatísticos utilizados para extrair conclusões acerca do problema de pesquisa proposto (Tabachnick \& Fidell, 2007). Utilizaram-se, ainda, a análise de modelagem estrutural composta por análise dos pressupostos estatísticos (outliers, lineariadade, normalidade e multicolinearidade), a análise da dimensionalidade, a validade dos construtos e o teste do modelo usando a técnica de PLS (Hair et al., 2010).

\section{Análise dos resultados}

A amostra foi composta por homens (52\%) e mulheres (48\%), com renda familiar entre $\mathrm{R} \$ 2.001$ e $6.000(46 \%)$ ou superior a $\mathrm{R} \$ 9.000$ (23\%), com faixa etária entre 19 e 35 anos (85\%), média de idade de 25 anos $(s=7,9)$, escolaridade predominante superior incompleto $(75 \%)$ e estado civil solteiro (87\%). As marcas que dominaram a pesquisa são Samsung e Apple, sendo que 68,3\% dos consumidores possuem essas marcas e responderam ao questionário sobre elas.

Considerando a contagem dos dados ausentes no banco de dados, foram encontrados 295 dados ausentes $(1,33 \%$ da base de 42 variáveis e 528 casos) dispersos em 86 questionários que foram excluídos, restando um total de 442 questionários completos. Notou-se um padrão aparente de concentração por falha na resposta, especialmente nas questões finais do instrumento. Aplicou-se o teste de aleatoriedade de dados ausentes (MCAR), que revelou a presença de um padrão significativo de perda de dados. Desse modo, preferiu-se eliminar os questionários com mais de $30 \%$ de dados ausentes, especialmente por estarem concentrados nas variáveis dependentes centrais do modelo, repondo os demais dados por meio do procedimento EM. Assim, foi possível sanar os dados ausentes na base de dados.

Os testes de normalidade mostraram uma tendência moderada de os dados apresentarem assimetria negativa, apesar de os valores não serem expressivos, isto é, os valores tendem a ser inferiores a 1 em módulo (Muthen \& Kaplan, 1992). Aplicando um teste formal dos parâmetros normais e avaliação geral do atendimento da normalidade (teste J-B), verificou-se que todas as variáveis violam o pressuposto de normalidade. Os problemas mais frequentes foram a curtose negativa (25 variáveis abaixo de -1) e, no geral, 27 variáveis apresentaram assimetria ou curtose fora dos limites de mais ou menos 1, o que indica um ponto de corte para o desvio da normalidade que não pode ser ignorado (Muthen \& Kaplan, 1992).

Nos estudos que utilizam técnicas de modelagem, usualmente é necessária a análise de correlações, que devem balizar os resultados quanto ao atendimento desse pressuposto. Isso decorre da constatação de que o coeficiente de Pearson é um índice de ajuste que só identifica respostas lineares entre as variáveis. Verificou-se que todas as variáveis apresentam correlações positivas e significativas, isto é, 861 correlações. Portanto, pode-se sugerir que as variáveis se ajustam de maneira relativamente linear (Hair et al., 2010).

Visando explorar as dimensões recorrentes em cada escala, empregou-se a Análise Fatorial Exploratória, conforme sugestão de Hair et al. (2010), Tabachnick e Fidel (2007). Na literatura, esta sugestão é premente: deve-se avaliar a dimensionalidade de um conjunto de dados. Na prática, seria necessário averiguar se os indicadores de cada construto partilham uma única faceta ou dimensão latente (Netemeyer, Bearden \& Sharma, 2003).Para esse fim, foi aplicada a extração por componentes principais (Tabela 1).

Os dados mostram que a AFE apresentou resultados satisfatórios em todos os indicadores definidos, destacando-se os valores elevados de variância explicada, o valor da medida KMO e comunalidades acima dos limites estabelecidos. Os resultados apontaram soluções unidimensionais em 
todos os casos, com comunalidades maiores que 0,500, variância explicada acima de 60\% e medida KMO (Kaiser-Meyer-Olsen) considerada "muito boa" (Hair, Black, Babin \& Anderson, 2010). Procedeu-se à avaliação da validade das medições utilizando, para isso, o modelo de equações simultâneas com variáveis latentes (Netemeyer, Bearden \& Sharma, 2003), aplicando-se como solução a estimação por Mínimos Quadrados Parciais (Partial Least Squares - PLS), sustentada pela pequena relação entre o número de parâmetros a serem estimados e o tamanho da amostra e devido aos expressivos desvios da normalidade (Hair et al., 2010). Desse modo, aceita-se uma boa evidência da unidimensionalidade dos construtos, reiterando a possibilidade de avançar na avaliação da fidedignidade das escalas de medição.

\begin{tabular}{l|l|l|l|l|l}
\hline Construtos & $\begin{array}{l}\text { Variância Ex- } \\
\text { plicada }\end{array}$ & Kmo & Teste Bartlet & GL & SIG. \\
\hline Confiança & $82 \%$ & 0,846 & 1681,00 & 6 & 0,00 \\
Comprometimento & $75 \%$ & 0,821 & 1292,17 & 6 & 0,00 \\
Lealdade & $79 \%$ & 0,921 & 3932,75 & 21 & 0,00 \\
Percepção Ética do consumidor & $78 \%$ & 0,938 & 4574,75 & 28 & 0,00 \\
\hline
\end{tabular}

Tabela 1 - Medidas gerais de adequação da solução fatorial dos construtos

Fonte: Dados da pesquisa.

Considerando tais pontos, para avaliar a validade convergente, verificaram-se as cargas fatoriais dos construtos. Sendo estas significativas no nível de $5 \%$ ou $1 \%$, têm-se evidências da validade convergente das medidas. Cabe notar que as estimativas de erro padrão utilizadas no PLS usam o bootstrapp como alternativa para estimar parâmetros e erros padrão. Assim, torna-se possível calcular erros padrão da estimativa do PLS, verificando a significância dos resultados.

A validade convergente verificou, como sugerem Bagozzi, Yi e Phillips (1991), que as cargas fatoriais dos indicadores foram significativas no nível de 1\%. Em sequência, foi avaliada a validade discriminante das escalas, analisando se a variância média extraída dos indicadores é inferior à variância compartilhada entre os construtos teóricos.

Também foram calculadas as medidas de confiabilidade composta (Composite Reliability - CC), variância média extraída (Average Variance Extracted - AVE) e Alpha de Cronbach (AC). Os resultados demonstram que houve validade discriminante, com correlações de elevadas a moderadas entre os construtos. Em termos de qualidade de mensuração, os dados revelaram boa adequação, dado que de Variância Média Extraída (VME ou AVE) superam a casa de 0,500 (Bollen, 1989) e as medidas de confiabilidade Alpha de Cronbach (AC) e Confiabilidade Composta (CC) superam a casa de 0,700.

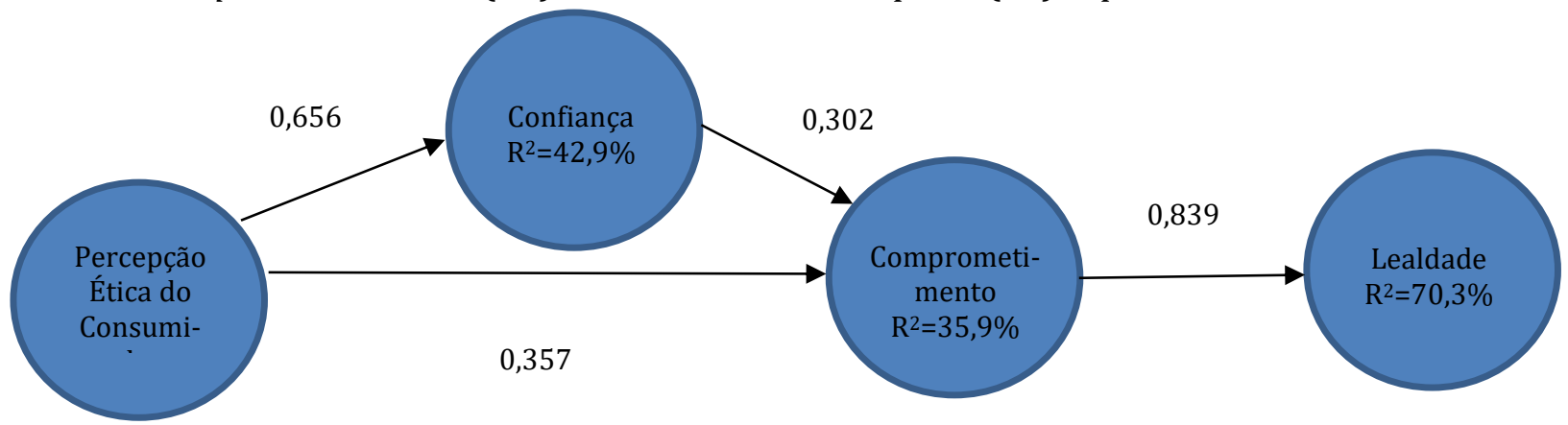

Figura 3 - Modelo de pesquisa: grupo ético

Fonte: Dados da pesquisa. Todos os pesos são significativos $(\mathrm{p}<0,05)$.

Os resultados da análise das hipóteses podem ser vistos na Tabela 2:

\begin{tabular}{|c|c|c|c|c|}
\hline Relação & Amostra & Erro & $\mathrm{t}$ & Sig. \\
\hline Percep. ética do consumidor -> Comprometimento & 0,357 & 0,0797 & 4,475 & $\mathrm{P}<0,01$ \\
\hline Percep. ética do consumidor $->$ Confianca & 0,656 & 0,0445 & 14.71 & $\mathrm{P}<0,01$ \\
\hline Comprometimento -> Lealdade & 0,839 & 0,0214 & 39,18 & $\mathrm{P}<0,01$ \\
\hline Confiança -> Comprometimento & 0,302 & 0,0890 & 3,369 & $\mathrm{P}<0,01$ \\
\hline
\end{tabular}

Tabela 2 - Resultado do teste de hipóteses do modelo.

Fonte: Dados da pesquisa. Observações: a) AMOSTRA é o peso padronizado obtido para amostra completa; b) 0 erro é o erro estimado da estimativa; c) 0 valor t é a razão entre o peso não padronizado pelo seu erro padrão. d) Sig. é significância da relação

\section{Discussão e implicações teóricas}

A questão da ética no relacionamento marca-consumidor faz-se presente, como indica Smith e 
Quelch (1996), que, em seus estudos, apuraram que o comportamento ético das marcas influencia a percepção e o comportamento do consumidor em relação às marcas. Os princípios da Ética em Marketing estão presentes na dinamicidade dessa relação. Eles influenciam o agir cotidiano da marca com o consumidor e vice-versa, à proporção que são reconhecidos como as regras e normas de conduta, as quais indicam aos parceiros o que é moralmente e virtuosamente aceito na relação, (Fonseca \& Souza Neto, 2007).

Desse modo, visando propor uma melhor compreensão da Lealdade e suas relações com a Percepção Ética do Consumidor, foi proposta esta pesquisa. Nesse sentido, buscaram-se evidências de um modelo integrativo de estágios de CBR, tendo como variável independente a Percepção Ética do Consumidor. Tal integração Ética-Marcas (CPE-CBR) foi estruturada em uma cadeia nomológica de antecedentes e consequentes do processo de desenvolvimento de relacionamentos entre consumidores e marcas. 0 trabalho também visa estender o conjunto de pesquisas em países emergentes sobre CPE - Consumer Perceived Ethicality (Brunk \& DeBoer, 2015), onde, segundo Leigh (2014), a questão ética tem especificidades, contribuindo para a validação da escala de mensuração bem como do conhecimento previamente consolidado sobre as relações entre teorias relacionadas à ética e relacionamento com marcas.

Os resultados deste trabalho indicam avanços no que se refere às questões éticas. Ao demonstrar um efeito significativo na confiança $\beta=0,656$, este estudo mostra que o perfil do consumidor nacional passa a incorporar outros aspectos para a decisão de compra. Kashmiri et al. (2017) destacava a necessidade de atenção ao comportamento ético das organizações diante da possibilidade de este impactar a decisão dos consumidores. 0 presente estudo corrobora empiricamente a observação desses autores ao demonstrar os efeitos das percepções éticas do consumidor em face das ações das organizações.

Outro avanço teórico que se pode inferir da presente pesquisa trata da relação entre lealdade e comprometimento, mediado pela confiança e percepção ética. McAlexander, Kim e Roberts (2015), Iglesias, Singh e Batista-Foguet (2011) indicavam a importância da relação do comprometimento e lealdade do consumidor como forma de valorização da marca. Ao se identificar que essa relação também é mediada pelos construtos confiança e percepção ética do consumidor, acrescentam-se novas variáveis com impactos significativos para aumento da lealdade.

Considera-se também uma contribuição acadêmica deste trabalho a aplicação e a validação da escala Consumer Perceived Ethicality (CPE) com a incorporação de dois novos itens (respeito ao consumidor e corrupção), justificados em razão de aspectos culturais (Arli \& Lasmono, 2009), ampliando a possibilidade de generalização da escala e sua replicação no contexto nacional. Esse acréscimo mostrouse adequado e corrobora a necessidade de adaptação da escala relatada no estudo de Leigh (2014).

Os resultados da pesquisa demonstram que a percepção ética do consumidor tem um efeito significativo $\beta=0,656$ na confiança, o que confirma que o comportamento ético da empresa é percebido positivamente pelo consumidor, o que aumenta sua confiança na marca, especialmente em smartphones. Esses resultados coadunam com os de Argoon e Rambocas (2011), Lin e Chuang (2016) e Chinomona (2013).

A percepção ética do consumidor e a confiança tiveram um efeito muito semelhante no comprometimento, $\beta=0,357$ e $\beta=0,302$, respectivamente. 0 comprometimento é o maior motivador para a lealdade, objeto desta pesquisa, porém a PEC tem um impacto significativo na confiança na marca, e a confiança é mediadora do comprometimento.

Nesse sentido, observa-se na cadeia nomológica que comprometimento afeta principalmente a confiança. 0 modelo foi capaz de explicar $70,3 \%$ da lealdade de consumidores estimulados por comportamentos éticos de empresas, o que contribui para sua robustez.

\section{Considerações finais}

As principais contribuições deste trabalho dão-se em três dimensões. Primeiramente, por explorar e descrever como o comportamento ético das organizações pode influenciar o comprometimento do consumidor com a marca, alterando sua lealdade. Este estudo apresenta novas descobertas no ciclo do relacionamento consumidor-marca e sua relação com as intenções comportamentais de consumidores; como o comportamento ético da empresa pode influenciar a lealdade do consumidor e que, no Brasil, houve a necessidade de inclusão de dois itens na escala PEC, corrupção e respeito ao consumidor.

0 segundo ponto refere-se à significativa relação entre comportamento ético e confiança, sendo esta mais afetada diretamente pelo comportamento ético e, possivelmente, pelo não ético. 0 comprometimento é um constructo e cunho mais estável que a confiança, sendo afetado direta e indiretamente. 0 
comprometimento é o principal constructo que afeta a lealdade, sendo esta a terceira dimensão mensurada e confirmada pela pesquisa.

Os resultados indicam ainda que o comportamento ético das empresas pode se apresentar como mais relevante pelos consumidores, considerando os resultados significativos dos caminhos do modelo. Isso sugere aos gestores de organizações implicações práticas, já que o comportamento indica a incorporação de atitudes mais críticas nos consumidores, o que leva as empresas a repensar atitudes e sua forma de atuação. Este estudo ainda indica uma necessidade de as organizações manterem padrões éticos em sua forma de atuação para manutenção de sua sobrevivência.

Como contribuição teórica, este trabalho utiliza e valida a escala PEC (Brunk, 2012) no Brasil, sugerindo acréscimo de dois itens (respeito ao consumidor e corrupção), ampliando a possibilidade de generalização da escala e sua replicação no contexto nacional. Tal resultado mostra diferenças culturais e demonstra a possiblidade de que a escala PEC possa ser utilizada de forma mais ampla na ciência, com maior poder explicativo, considerando suas propriedades psicométricas e o número reduzido de itens que possui, o que amplia as possibilidades de sua aplicação, sendo esta uma contribuição acadêmica.

Como contribuições práticas, esta pesquisa mostra o perfil dos consumidores de smartphones com uma mudança de posicionamento, já que atentam nas questões relativas a ambientes, condições de trabalho, relacionamento com o consumidor e também nas questões éticas envolvendo a organização. Essa preocupação deve ser objeto de atenção por parte de gestores, pois consumidores não tomam decisão de compra baseados apenas em variáveis ligadas a preço e qualidade, também aspectos éticos fazem parte da análise feita por eles. Esses achados também demonstram a necessidade de as empresas pautarem seus negócios e ações de forma ética, pois o comprometimento da imagem da organização com negócios antiéticos pode levar à perda de participação no mercado. A confiança é significativamente afetada pela percepção ética, apresentando aos gerentes uma afirmação de que suas ações são acompanhadas de perto pelos consumidores e que eles devem adotar medidas rápidas para conter os eventuais problemas de uma percepção não ética. A empresa deve ser sincera com o cliente, responder às falhas rapidamente. Assim, a confiança na marca não será abalada e, consequentemente, também não sofrerá abalo o comprometimento com a marca.

No que se refere a prática, se observa relevantes contribuições para profissionais de administração, executivos empresariais e gestores públicos, bem como acadêmicos da área. Primeiramente, os resultados provam que as percepções éticas do consumidor afetam de forma significativa a confiança na empresa. No Brasil, parece que está prática muitas vezes faz parte do discurso das empresas, porém não se efetiva em suas ações. Recentemente pode-se verificar o caso relevante da Companhia Vale do Rio Doce. Em março de 2019 toda a Diretoria e Presidência da Vale foi demitida. Segundo Setti e Cavalcanti (2019): “O novo presidente terá ainda de atuar na recuperação da credibilidade da mineradora no mercado, que já perdeu o grau de investimento - espécie de selo de bom pagador - concedido pela agência de classificação de risco Moody's. Para analistas, o rebaixamento pode ser acompanhado pelas concorrentes Fitch e S\&P, o que pode levar investidores institucionais, sobretudo estrangeiros, a ter de vender as ações. Desde a tragédia, em 25 de janeiro, a Vale já perdeu quase R 50 bilhões em valor de mercado". Outros casos conhecidos e recentes dizem respeito a Petrobrás e Construtora Odebrecth. No caso da construtora, os eventos foram de tal seriedade que a empresa mudou o nome, cores e logotipo. Casos em mercados de consumo e ética, em especial envolvendo trabalho escravo ou infantil, estão relacionados a queda de credibilidade de marcas de moda internacional com fabricas no exterior e no país, bem como os maiores fabricantes de chocolate do mundo (Correio Braziliense, 2016). Empresas como a Petrobrás, que possuem gestão pública, também têm sido afetadas por práticas administrativas danosas, que deveriam ser evitadas, com consequências relevantes para empresa e sua credibilidade. Segundo o UOL (2016): "Dois anos após o começo da operação Lava Jato, muita coisa mudou na estatal. Executivos foram presos e a empresa enfrenta processos na Justiça; as ações caíram e a petroleira passou a valer menos, e sua dívida, que já era grande, aumentou ainda mais. Mas, certamente, a Lava Jato marcou o início de uma das piores fases já vistas nos mais de 60 anos da Petrobras". Práticas que levam a prisão talvez sejam um extremo da administração de empresas. Por outro lado, os gestores devem estar atentos aos impactos positivos das ações Éticas (e negativos caso ocorram problemas) no comprometimento dos consumidores com estas marcas, pois o mesmo é o maior antecedente da lealdade dos consumidores. Pesquisas anteriores como Creyer (1997) apontam nesta direção, porém com menor ênfase. Neste sentido, o estudo aqui apresentado contribui e aponta para uma análise mais precisa, pois trata de amostra mais recente indicando que as gerações atuais dão ainda maior importância a ética das empresas 
para definirem seus comportamentos. Uma pesquisa realizada pela Nielsen (2015) em 60 países, mostra que $66 \%$ dos entrevistados globais dizem que estão dispostos a pagar mais por produtos e serviços que vêm de empresas que estão comprometidas com um impacto social, ético e ambiental positivo, sendo que este valor era de 55\% em 2014, e 50\% em 2013. Isso sugere práticas pelos gestores de marketing mais personalizadas e éticas para vender, desenvolver e manter Relacionamentos Consumidor-Marca (Kaylene \& Robert, 2011; Goldmansachs, 2017).

Uma outra contribuição deste trabalho para gestores se refere às dimensões éticas valorizadas pelos consumidores brasileiros. Todas as dimensões apresentaram impacto na percepção ética das empresas pelos consumidores, sendo a ordem de importância: (1) ser socialmente responsável; (2) cumprir leis; (3) respeitar normas morais; (4) respeitar seus consumidores; (5) não aceitar corrupção; (6) não aceitar agredir o ambiente; (7) só tomar as decisões considerando todas as consequências para os envolvidos e (8) ter produtos de qualidade. Com pesos altos e similares nas primeiras sete dimensões (entre $0,82630,926$ ), este trabalho indica aos gestores as linhas práticas para trabalhar as percepções dos consumidores sobre comportamento ético de suas empresas. Este trabalho sugere ainda a inclusão de duas novas dimensões significativas no cenário brasileiro na escala internacional de percepção ética PEC (itens 4 e 5). Esta contribuição indica a necessidade de uma escala internacional mais generalizável em países do terceiro mundo, onde desrespeito ao consumidor e corrupção podem ser práticas mais observadas e que impactam as intenções e comportamentos dos consumidores e do mercado, constituindo também importante contribuição acadêmica.

A limitação da pesquisa concentra-se em não abranger pessoas de outras gerações que utilizam smartphones e também mensurar outros produtos de consumo como computadores, consoles de jogos dentro do universo da TI. Para pesquisas futuras, sugerem-se estudos de casos com a mensuração da escala CPE, pois acontecimentos recentes em Minas Gerais, como os desastres de Brumadinho e Mariana, podem influenciar a percepção ética do consumidor em relação às empresas e marcas, conforme foi comprovado nesta pesquisa.

Pela relevância do tema "ética empresarial" nos dias de hoje e pela comprovação de sua importância para a amostra (eminentemente jovem), sugere-se que as organizações devem ter mais atenção com a ética nos negócios como antecedente de resultados e relacionamentos de longo prazo com consumidores no futuro.

\section{Referências}

Arjoon, S.; Rambocas, M. (2011). Ethics and Customer Loyalty: Some Insights into Online Retailing Services. International Journal of Business and Social Science, 2(14), 135-142.

Arli, Denni \& K. Lasmono, Hari. (2009). Consumers' perception of corporate social responsibility in a developing country. International Journal of Consumer Studies. 34.46 - 51.

Auger, P., Devinney, T.M., Louviere, J.J., \& Burke, P.F. (2010). The importance of social product attributes in consumer purchasing decisions: A multi-country comparative study. International Business Review, 19(2), 140-159.

Bagozzi, R.P.; Yi, Y.; Phillips, L.W. (1991). Assessing Construct Validity in Organizational Research. Administrative Science Quarterly, 36 (3), 421.

Becchetti, L., \& Costantino, M. (2010). Fair trade in Italy: Too much 'movement' in the shop? Journal of Business Ethics, 92(2), 181-203.

Besiou, M.; Hunter, M. \& Wassenhove, L. (2013), A Web of Watchdogs: Stakeholder Media Networks and Agenda-Setting in Response to Corporate Initiatives, Journal of Business Ethics, 118, (4), 709-729

Blackston, M. (1992). Observations: Building Brand Equity by Managing the Brand's Relationships. Journal of Advertising Research, 32 (3), 79-83.

Bloemer, J.M.M., \& Odekerken-Schröder, G. (2002). Store satisfaction and are loyalty explained by customer and store-related factors. Journal of consumer satisfaction, dissatisfaction and complaining behavior, 15, 68-80.

Bollen, K.A. (1989). Structural equations with latent variables. New York: Wiley.

Bowden, J.H. (2009). Customer engagement: A framework for assessing customer-brand relationships: The case of the restaurant industry. Journal of Hospitality Marketing \& Management, 18 (6), 574- 596.

Brunk, K.H. (2010). Exploring origins of ethical company/brand perceptions - A consumer perspective of corporate ethics. Journal of Business Research, 63(3), 255-262.

Brunk, K.H., \& Blümelhuber, C. (2011). One strike and you're out: Qualitative insights into the formation 
of consumers' ethical company or brand perceptions. Journal of Business Research, 64(2), 134-141.

Brunk, K.H. (2012). Un/ethical Company and Brand Perceptions: Conceptualizing and Operationalizing Consumer Meanings. Journal of Business Ethics, 111(4), 551-565.

Brunk, K.H. \& DeBoer, C. (2015) Ethical Brand Perception Formation When Information Is Inconsistent - an Impression Formation Perspective, in NA - Advances in Consumer Research Volume 43, eds. Kristin Diehl and Carolyn Yoon, Duluth, MN : Association for Consumer Research, Pages: 319-323.

Callicott, J.B. \& Frodeman, R. (2009). Encyclopedia of environmental ethics and philosophy. United States of America: Gale, Cengage Learning.

Carrigan, M., \& Attalla, A. (2001). The myth of the ethical consumer - Do ethics matter in purchase behavior? Journal of Consumer Marketing, 18(7), 560-577.

Carrigan, M., Szmigin, I., \& Wright, J. (2004). Shopping for a better world? An interpretive study of the potential for ethical consumption within the older market. Journal of Consumer Marketing, 21(6), 401417.

Carrigan, M., Moraes, C., \& Leek, S. (2011). Fostering responsible communities: A community social marketing approach to sustainable living. Journal of Business Ethics, 100(3), 515-534.

Carroll, B.A. \& Ahuvia, A. C. (2006). Some Antecedents and Outcomes of Brand Love. Marketing Letter, 17, 79-89.

Chabowski, B.R., Mena, J.A., \& Gonzalez-Padron, T.L. (2011). The structure of sustainability research in marketing, 1958-2008: a basis for future research opportunities. Journal of the Academy of Marketing Science, 39, 55-70.

Chaudhuri, A. \& Hoolbrook, M.B. (2002). Product-class effects on brand commitment and brand outcomes: The role of brand trust and brand effect. Journal of Brand Management, 10(1), 33-58.

Chinomona, R. (2013). The Influence of Brand Experience on Brand Satisfaction, Trust and Attachment in South Asia. International Business \& Economics Research Journal, 12 (10), 1303-1316.

Chiu, C.M., Huang, H.Y. \& Yen, C.H. (2010). Antecedents of online trust in online auctions. Electronic Commerce Research and Application, 9, 148-159.

Correio Braziliense (2016). Após denúncia de uso de mão de obra infantil, Nestlé e Hershey se defendem.

Correio Braziliense edição de 04/03/2016. Disponível em https://www.correiobraziliense.com.br/app/noticia/mundo/2016/03/02/interna_mundo,520225/apos-denuncia-deuso-de-mao-de-obra-infantil-nestle-e-hershey-se-defe.shtml. Acesso em 26/05/2019

Creyer, E. H. (1997). The influence of firm behavior on purchase intention: do consumers really care about business ethics?, Journal of Consumer Marketing, 14 (6), 421-432.

Cuza, A.I. (2012). Motivation in Insurance Sales, University Annals, Economic Sciences Series. 12 (1), $437-536$.

Das, G.; Agarwal, J.; Malhotra, N.K. \& Varshneya, G., (2019), Does brand experience translate into brand commitment? A mediated-moderation model of brand passion and perceived brand ethicality, Journal of Business Research, 95, (C), 479-490

Dennis, C. et al. (2016). The role of brand attachment strength in higher education. Journal of Business Research,v. 69, 3049-3057.

Dick, A.S. \& Basu, K. (1994). Customer loyalty: Toward an integrated conceptual framework. Journal of Academy of Marketing Science, 22, 99-113.

Dib H., \& Alhaddad, A. (2014). The Hierarchical Relationship between Brand Equity Dimensions, European Scientific Journal, 10 (28), 183-194.

Dutt, A.K. \& Wilber, C.K. (2010). Economics and Ethics. Great Britain: Palgrave Macmillan.

Eisingerich, A.B. \& Rubera, G. (2010). Drivers on Brand Commitment: A Cross-National Investigation. Journal of International Marketing, 18 (2), 64-79.

Esch, F.R., Langner, T., Schmitt, B.H. \& Geus, P. (2006). Are brands forever? How brand knowledge and relationships affect current and future purchases. The Journal of Product and Brand Management, 15 (2), 98-105.

Fan, Y. (2005). Ethical branding and corporate reputation. Corporate Communications: An International Journal, 10(4), 341-350.

Fetscherin, M., \& Heilmann, T. (2015). Brand Relationship Rule. In: Fetscherin, M., \& Heilmann, T. (Eds) Consumer Brand Relationships: Meaning, Measuring, Managing. New York: Palgrave Macmillan.

Folkes, V.S., \& Kamins, M. A. (1999). Effects of information about firms' ethical and unethical actions on consumers' attitudes. Journal of Consumer Psychology, 8(3), 243-259. 
Fonseca, F.R.B. \& Souza Neto, A.F. de. (2007). A ética em marketing aplicada no relacionamento marcaconsumidor. XXXI Encontro da ANPAD, Rio de Janeiro, de 22 a 26 setembro, 2007

Fournier, S. (1998). Consumers and their Brands: Developing Relationship Theory in: Consumer Research. Journal of Consumer Research, 24, 343-373.

Ganesan, S. (1994). Determinants of Long-Term Orientation in Buyer-Seller Relationships, Journal of Marketing, 58 (2), 1-19.

Goldmansachs (2017). Millennials Coming out of Age. Available at http://www.goldmansachs.com/ourthinking/pages/millennials/.

Grohmann, B. \& Bodur, H. (2015), Brand Social Responsibility: Conceptualization, Measurement, and Outcomes, Journal of Business Ethics, 131, (2), 375-399

Hair, J.F., Black W.C., Babin, B.J., \& Anderson, R.E. (2010) Multivariate Data Analysis. $7^{\text {th }}$ ed. Harlow: Pearson Education Limited.

Hamidizadeh, M.R., Alavije, M. R. K., \& Rezaee, M. (2014) The effect of perceived business ethics on brand personality dimensions e creation of brand equity in developing countries. Central European Business Review, 3(3), 46-53.

He, H., Li, Y., \& Harris, L. (2012). Social identity perspective on brand loyalty. Journal of Business Research, $65(5), 648-657$.

Hur, W.M.; Park, J. \& Kim, M. (2010). The role of commitment on the customer benefits- loyalty relationship in mobile service industry. The Service Industries Journal, 30, 2293-2309.

Hess, J., \& Story, J. (2005) Trust-based commitment: multidimensional consumer-brand relationships. Journal of Consumer Marketing, 22 (6), 313-322.

Hutchinson, D., Singh, J., Svensson, G., \& Mysen, T. (2013). Towards a model of conscientious corporate brands: A Canadian study. Journal of Business and Industrial Marketing, 28(8), 687-695.

Iglesias, 0.; Singh, J.J. \& Batista-Foguet, J.M., (2011). The role of brand experience and affective commitment in determining brand loyalty. Journal of Brand Management. 18 (8), 570-582.

Ingram R., \& Skinner, S.J., (2005). Consumer evaluation of unethical marketing behaviors: the role of customer commitment. Journal of Business Ethics, 62 (3), p. 237-252.

Jang, H. et al. (2014). The influence of on-line brand community characteristics on community commitment and brand loyalty. International Journal of Eletreonic Commerce, 12, 57-80.

Kashmiri, S., Nicol, C.D., \& Hsu, L. (2017). Birds of a feather: intraindustry spillover of the Target customer data breach and the shielding role of IT, marketing, and CSR. Journal of the Academy of Marketing Science, 45(2), 1-21.

Kaylene, C. W., \& Robert, A. P. (2011). Marketing to the generations. Journal of Behavioral Studies in Business, 3, 1-17.

Keller, K. L. (1993) Conceptualizing, measuring and managing customer-based brand equity. Journal of Marketing, 57 (1), 1-22.

Keller, K. L. (2001). Building customer based brand equity: A blueprint for creating strong brands. (Working Paper 1-107), Cambridge: Marketing Sciences Institute.

Knapp, H. L., \& Taylor, E. H. (1994). Commitment and its Communication in romantic relations. In Weber, A. L.; Harvey, J. A. (Eds.). Perspectives on close relationship. Needham Heights: Simon \& Schuster, 153175.

Laroche, M., Bergeron, J., \& Barbaro-Forleo, G. (2001). Targeting consumers who are willing to pay more for environmentally friendly products. Journal of Consumer Marketing, 18(6), 503-520.

Leigh. A. (2014) Emerging markets and their ethical challenge. Ethical Leadership. Available at http://www.ethical-leadership.co.uk/emerging-markets/. Access on 19 Sept. 2017.

Lin, C.P., \& Chuang, C.M. (2016). Corruption and Brand Value. International Marketing Review, 33(6), 758-780.

Malhotra, N.K. (2001) Self-Concept and Product Choice: an Integrated Perspective. Journal of Economic Psychology, 9, 1-28.

Mcalexander, J. H.; Kim, S. K.; Roberts, S. D. (2015). Loyalty: The Influences of Satisfaction and Brand Community Integration. Journal of Marketing Theory and Practice. 1-11.

Megicks, P., Memery, J., \& Williams, J. (2008). Influences on ethical and socially responsible shopping: Evidence from the UK grocery sector. Journal of Marketing Management, 24(5-6), 637-659.

Morgan, R. M. \& Hunt S. D. (1994). The Commitment- Trust Theory of Relationship Marketing. Journal of Marketing, 58 (3), 20-38. 
Muthen, B; kaplan, D. (1992). A comparison of some methodologies for the factor analysis of non-normal Likert variables: A note on the size of the model. British Journal of Mathematical and Statistical Psychology, 45 (10, 19-30.

Netemeyer, R. G., Bearden, W. O. \& Sharma, S. (2003). Scaling procedures: issues and applications. New York, USA: Sage Publications.

Nielsen. (2015). Escolhas sustentáveis: como empresas socialmente responsáveis lucram com isso?. São Paulo/SP. Disponível em: http://www.nielsen.com/br/pt/insights/news/2015/Escolhas-sustentaveisComo-empresas-socialmente-responsaveis-lucram-com-isso.html. Acesso em 23 mai. de 2019.

Nga, J. K. H.; Soo, N. W. M. (2013). A study on the factors influencing the perception of ethical branding among gerneration y consumers in Malaysia. AFBE Journal, 6 (1), 1-40.

O'Connor, C. (2014). Shoppers use app to boycott Israel in grocery store aisles. Forbes 8/06/2014 Available at: http://www.forbes.com/sites/clareoconnor/2014/08/06/shoppersuse-

app-to-boycott-israel-in-grocery-store-aisles/.

Oliver, R. L. (1999). Whence consumer loyalty? Journal of Marketing, 63 (Special Issue), 33-44.

Parvatiyar, A. \& Sheth, J. (2000). The domain and conceptual foundations of relationship marketing. In Sheth, J., \& Parvatiyar, A. Handbook of relationship marketing. Thousand Oaks: Sage.

Petzer, D. Mostert, P., Kruger, L. \& Kuhn, S. (2014). The dimensions of brand romance as predictors of brand loyalty among cell phone users. South African Journal of Economic and Management Sciences, v. 17 (4), 457-470.

Pojman, L.P. \& Fieser, J. (2012). Ethics: Discovering Right and Wrong. 7th Ed. Boston: Cengage Learning. Quinn, M.J. (2013). Ethics for the Information Age. 5th Ed. Upper Saddle River: Pearson Education.

Raju, Sekar; Unnava, H. R., \& Montgomery, N. (2009). The Effect of Brand Commitment on the Evaluation of Nonpreferred Brands: A Disconfirmation Process, Journal of Consumer Research, 35 (February), 851863.

Rindell, A., Svensson, G., Mysen, T., Billstro"m, A., \& Wile'n, K. (2011). Towards a conceptual foundation of 'Conscientious Corporate Brands'. Journal of Brand Management, 18(9), 709-719.

Schmalz, S., \& Orth, U.R. (2012). Brand Attachment and Consumer Emotional Response to Unethical Firm Behavior. Psychology \& Marketing, 29(11), 869-884.

Setti R., \& Cavalcanti, G. (2019) Novo presidente da Vale terá o desafio de recuperar confiança do mercado na empresa. Jornal $O$ Globo Edição de 04/03/19. Acesso em 26/05/2019. Disponível em https://oglobo.globo.com/economia/novo-presidente-da-vale-tera-desafio-de-recuperar-confiancado-mercado-na-empresa-23497075. Acesso em 26/05/2019

Shanahan, K. J., \& Hyman, M. R. (2003). The development of a virtue ethics scale. Journal of Business Ethics, 42(2), 197-208.

Shaw, D. (2007). Consumer voters in imagined communities. International Journal of Sociology and Social Policy, 27 (3/4), 135-150.

Sierra, V., Iglesias, O., Markovic, S., \& Singh, J.J. (2017) Does Ethical Image Build Equity in Corporate Services Brands? The Influence of Customer Perceived Ethicality on Affect, Perceived Quality, and Equity. Journal of Business Ethics, 144, 661-676.

Singhapakdi, A., Gopinath, M., Marta, J.K., \& Carter, L.L. (2008). Antecedents and consequences of perceived importance of ethics in marketing situations: A study of Thai businesspeople. Journal of Business Ethics, 81(4), 887-904.

Singh, Jatinder J., Oriol Iglesias, \& Joan Manuel Batista (2012), Does Having an Ethical Brand Matter? The Influence of Consumer Perceived Ethicality on Trust, Affect and Loyalty. Journal of Bunisess Ethics 111, 541-549.

Smith, N. C., \& Quelch, J. A (1996). Ethics in Marketing, Irwin, Boston, MA.

Sondoh Jr, S. L., Omar, M. W., Wahid, N. A., Ismail, I., \& Harun, A. (2007). The effect of brand image on overall satisfaction and loyalty intention in the context of color cosmetic. Asian Academy of Management, 12(1), 83-107.

Sreejesh, S. (2014) Consumers' attitudinal and behavioral ties with brands: an integrative approach to build a consumer-brand relationship model. Journal of Research for Consumers, 1 (26), 32-75.

Sreejesh, S. \& Roy, S. (2015). A New Consumer Brand Relationships Framework. In Fetscherin, M.; \& Heilmann, T. (2015), Consumer Brand Relationships. London: Palgrave Macmillan, 165-197.

Strauss, A. \& Corbin, J. (1994). Grounded Theory Methodology. In Denzin, N.K., \& Lincoln Y.S. (Eds.) Handbook of Qualitative Research. Thousand Oaks: Sage Publications, 217-285. 
Tabachnick, B., \& fidell, L. (2007). Using Multivariate Statistics. 5 ed. Boston: Pearson, 2007.

Wilkie, W.L., \& Moore, E.S. (2012). Expanding our Understanding of Marketing in Society. Journal of the Academy of Marketing Science, 40, 53-73.

Wu, S.I., \& Chen, J.H. (2015). The Influence of CSR on Brand Relevant Aspects. Journal of Management and Sustainability, 5(3), 17-30.

Young, K.M,. \& Hee, L.S. (2006). The Effects of Corporate Social Responsibility on Brand Attachment and Brand Equity. The Research Journal of the Costume Culture, 14 (4), 2006, 684-697.

Zhang, N. et al. (2013). How do different types of community commitment influence brand commitment? The mediation on brand attachment. Cyberpsychology Behavior and Social Networking 16(11), 836-843. 